\section{Búsqueda activa de enfermedad celíaca en familiares de primer grado de casos celíacos}

\author{
MARCELA BEJARES ${ }^{1, a}$, AMAYA OYARZÚN², \\ YALDA LUCERO $^{3, \mathrm{a}, \mathrm{b}}$, NELLY ESPINOZA ${ }^{4}$, \\ KARLA BASCUÑÁN ${ }^{5, \mathrm{c}}$, MAGDALENA ARAYA ${ }^{2}$
}

\section{Active search of celiac disease among first degree relatives of known celiac patients}

Background: Active search of celiac disease (CD) among risk groups has significantly increased the scope of known clinical variants. Aim: To measure the frequency and clinical characteristics of $C D$ among first degree relatives (FDR) of known celiac cases. Material and Methods: Between January 2012-August 2013, 37 patients with celiac disease brought 113 FDR for assessment. Their clinical data was recorded and a blood sample was obtained to measure serum Immunoglobulin A (IgA) levels, anti-transglutaminase ( $t T G)$ and anti-endomisial (EMA) antibodies. Cases with positive serology were advised to have an intestinal biopsy. Results: Fourteen relatives (12.4\%) had positive serological results and none had IgA deficiency. Among IgA-tTG (-) cases, measurement of IgA/IgG-tTG identified an additional case. Two of the 14 relatives were EMA positive. All 14 cases were advised to have an intestinal biopsy, but only 6 accepted the procedure. In two, the intestinal lesion was classified Marsh $\geq 2$ and active $C D$ was diagnosed. Histology in the remaining four was Marsh 0/1 and were diagnosed potential CD, remaining under control, without gluten free diet. Conclusions: Serological prevalence of CD among first degree relatives of known celiac cases was 15 fold greater than in THE general Chilean population, strongly supporting the idea of implementing active search to customary clinical practice. Determination of IgA/IgG-tTG may be useful to improve the yield of active search. Intestinal biopsies were crucial to differentiate active classic CD from potential CD.

(Rev Med Chile 2015; 143: 1521-1526)

Key words: Celiac Disease; Immunoglobulin A; Transglutaminase 2, human.

\author{
'Departamento de Pediatría Occidente, \\ Escuela de Medicina, Universidad de \\ Chile. Servicio de Pediatría, Hospital \\ San Juan de Dios. Santiago de Chile, \\ Chile. \\ ${ }^{2}$ Laboratorio de Inmunogenética, INTA, \\ Universidad de Chile. Santiago de \\ Chile, Chile. \\ ${ }^{3}$ Departamento de Pediatría Oriente, \\ Escuela de Medicina, Universidad de \\ Chile. Servicio de Pediatría, Hospital \\ Luis Calvo Mackenna, Santiago de \\ Chile, Chile. \\ ${ }^{4}$ Unidad de Gastroenterología Infantil, \\ Hospital Militar. Santiago de Chile, \\ Chile. \\ apediatra, becada del programa \\ de subespecialización en \\ Gastroenterología y Nutrición Escuela \\ de Postgrado Facultad de Medicina, \\ Universidad de Chile. \\ 'Pediatra, PhD. \\ 'Nutricionista, candidata a Doctor en \\ Nutrición, Programa Universidad de \\ Chile. \\ Financiamiento: Este estudio fue \\ financiado por la Corporación para \\ Apoyo de la Investigación Científica \\ en Nutrición (Cinut), Grifols $®$ \\ y Biodiagene ${ }^{\circledR}$. Las entidades \\ financiadoras no tuvieron influencia \\ alguna en el diseño del estudio; en la \\ recolección, análisis o interpretación de \\ los datos; en la preparación, revisión ni \\ aprobación del manuscrito. \\ Recibido el 2 de diciembre de 2014, \\ aceptado el 20 de agosto de 2015. \\ Correspondencia: \\ Prof. Magdalena Araya \\ INTA, Universidad de Chile \\ El Líbano 5524, Macul-Santiago, Chile. \\ Teléfono: 56229781411 \\ maraya@inta.uchile.cl
}

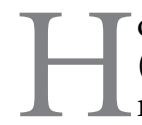
oy entendemos la enfermedad celíaca (EC) como un desorden multisistémico mediado inmunológicamente, provocado por el gluten y prolaminas relacionadas, en individuos genéticamente susceptibles. Se caracteriza por la presencia de una combinación variable de síntomas digestivos y extradigestivos, especialmente aquellos asociados a procesos autoinmunes, junto a la presencia de anticuerpos específicos (anticuerpos antiendomisio [EMA] o antitransglutaminasa [tTG]) en sangre) y a algún grado de daño en la mucosa del intestino delgado.

Contrario a lo que se pensaba hasta hace una o dos décadas, los estudios de tamizaje en población sana o de riesgo han mostrado que la EC es altamente prevalente, con frecuencias de 0,5 a $1 \%$. En Chile, la segunda Encuesta Nacional de Salud (2009-2010) evaluó una muestra representativa de 
los mayores de 15 años de todo el país, encontrando que $0,75 \%$ de los individuos poseía anticuerpos tTG2 en el plasma. Asimismo, la condición fue más frecuente en mujeres y en edades mayores. La prevalencia confirmada por biopsia intestinal actualmente se estima en $0,6 \%$.

Clínicamente, la EC se manifiesta de manera muy variable, desde cuadros asintomáticos u oligo-sintomáticos, hasta la típica diarrea crónica con desnutrición descrita clásicamente. La falta de sospecha clínica condiciona, en una proporción importante de los casos, el no hacer o retardar significativamente el diagnóstico. La evidencia actual sugiere que el tiempo de exposición al gluten aumenta el riesgo de desarrollar procesos autoinmunes. Los individuos con presentaciones atípicas serían tan susceptibles a presentar complicaciones por déficit y autoinmunidad de la enfermedad como aquellos que presentan el cuadro florido. Por esto es que hoy se considera necesario realizar una "búsqueda activa" en los grupos de mayor riesgo, es decir, estudiar a los individuos según presenten patologías definidas como "de riesgo", sin esperar que desarrollen síntomas y consulten por ellos. Estos casos, que si no son buscados permanecen sin diagnosticar, se benefician también y claramente con el tratamiento de la dieta sin gluten. Dentro de los llamados grupos de riesgo, destacan los familiares de primer grado de celíacos (FPG) ya diagnosticados. En ellos se describe una prevalencia entre 2,6 y 16\%, calculándose un riesgo 5 a 20 veces mayor que en la población general. El riesgo es aún mayor en familiares portadores de HLA DQ2 o DQ8. El tratamiento con dieta libre de gluten es efectiva en la gran mayoría de los casos, la sintomatología y la lesión histológica remiten, se negativiza la serología y se ha estimado que los costos en salud del paciente disminuyen en $40 \%$. Todo ello mejora la calidad de vida del paciente y contribuye a aminorar la carga en salud que representa la enfermedad y a evitar la mayor mortalidad que tienen los pacientes con EC.

La "búsqueda activa" recomendada hoy para los grupos de riesgo de EC ha ampliado significativamente el rango de variantes clínicas atípicas de la EC, haciendo claramente necesario que se hagan más estudios que las caractericen correctamente. La literatura sobre este tipo de pacientes aún es escasa y en nuestro medio no hay datos, por lo que en este estudio el objetivo se definió en determinar la frecuencia de detección de EC en FPG de pacientes ya diagnosticados, y caracterizar a estos casos desde el punto de vista clínico.

\section{Pacientes y Método}

\section{Sujetos}

Este es un estudio descriptivo, observacional, realizado entre enero de 2012 y agosto de 2013, que incluyó a los casos de celíacos diagnosticados según los criterios de ESPGHAN (2012), incluyendo la presencia de anticuerpos EMA tTG2, o ambos y una biopsia de intestino delgado con lesión Marsh 2 o más, y sus familiares directos (padres, hermanos), que participaban en la Corporación de Apoyo a los Celíacos (COACEL) o eran controlados en CEDINTA (Centro Diagnóstico del Instituto de Nutrición y Tecnología de los alimentos, INTA, Universidad de Chile). El protocolo de estudio fue aceptado por el Comité de Ética para la investigación en seres humanos del INTA, Universidad de Chile.

Se excluyeron aquellas familias en las que no se evaluó al grupo familiar completo. La información se recogió en un formulario pre-diseñado, durante una entrevista semi-estructurada, en la que se registraron datos demográficos, historia de salud personal y de los familiares, incluyendo síntomas digestivos y extradigestivos.

\section{Evaluación del estado nutricional}

Todos los sujetos fueron pesados y medidos, con una balanza digital y estadiómetro (SECA) de precisión $(0,1 \mathrm{~kg}$ y $0,1 \mathrm{~cm}$, respectivamente) en posición de Frankfurt, con la menor cantidad de ropa posible y descalzos.

\section{Procedimientos}

Se obtuvo una muestra de sangre de $5 \mathrm{ml}$, de la vena anticubital, en un tubo con EDTA, fue recolectada de cada sujeto de estudio. Las muestras se centrifugaron a $3.000 \mathrm{rpm}$ por $15 \mathrm{~min}$ y se almacenaron a $-20^{\circ} \mathrm{C}$ hasta su procesamiento en el INTA. La determinación de IgA total se realizó en una alícuota de suero mediante un test de ELISA comercial $\left(\right.$ ALPCO $\left.^{\circledR}\right)$. Se consideró normal un valor de IgA total menor o igual a140 $\mathrm{mg} / \mathrm{dL}$ en adultos y mayor o igual $21 \mathrm{mg} / \mathrm{dL}$ en niños, de acuerdo a lo sugerido por el fabricante, para las edades incluidas en el protocolo. La tTG2 se midió mediante dos métodos de ELISA: i) un 
kit comercial (AEUSKU ${ }^{\circledR}$, Finlandia) que mide IgA-tTG2, y un kit comercial que determina IgA/ IgG- tTG (AEUSKU ${ }^{\circledR}$, Finlandia); en ambos casos se siguieron las instrucciones del fabricante, considerando positivo un valor de IgA-tTG2 $\geq 19 \mathrm{UI} / \mathrm{ml}$ y de IgA/IgG anti-tTG2 $\geq 25 \mathrm{UI} / \mathrm{ml}$. La presencia de anticuerpos EMA se estudió mediante inmunofluorescencia indirecta, en cortes de esófago de mono $\left(\mathrm{IMMCO}^{\circledR}\right)$.

\section{Análisis estadístico}

Las variables categóricas fueron comparadas entre grupos mediante test de $\chi^{2}$ o test exacto de cuando fue apropiado, y las variables continuas mediante t-Student o ANOVA, según su distribución. Para el análisis se utilizó Epi Info ${ }^{\mathrm{TM}} 7$ (CDC, USA), considerando significativo un valor $\mathrm{p}<0,05$.

\section{Resultados}

Se reclutaron 37 casos celíacos, que aportaron 113 familiares de primer grado (Figura 1), cuya edad, sexo, estado nutricional y sintomatología se muestran en la Tabla 1. No se encontraron diferencias en edad, estado nutricional o género entre los familiares de primer grado con serología positiva y negativa (Tabla 1). De los síntomas digestivos encontrados, sólo la distensión abdominal mostró una frecuencia significativamente mayor en familiares con serología positiva ( $\mathrm{p}<0,04$, $\left.\chi^{2}\right)$. La IgA sérica estaba por debajo de los valores normales en 10 casos $(6,6 \%)$.

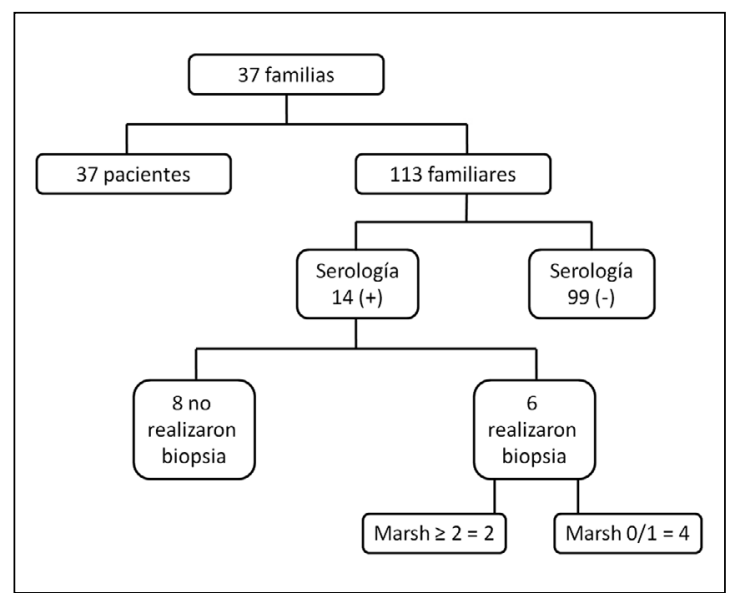

Figura 1. Algoritmo.

Tabla 1. Edad, sexo, estado nutricional y sintomatología en familiares de primer grado de pacientes celíacos

\begin{tabular}{|c|c|c|c|}
\hline & $\begin{array}{l}\text { Familiares serología (+) } \\
\qquad(\mathrm{n}=14)\end{array}$ & $\begin{array}{l}\text { Familiares serología (-) } \\
\qquad(n=99)\end{array}$ & $\begin{array}{l}\mathbf{p} \\
\chi^{2}\end{array}$ \\
\hline Edad promedio (rango) & $33,6(4,2-66,3)$ & $36,1(1,2-73,0)$ & \\
\hline Mujeres n (\%) & $8(57,1)$ & $53(53,5)$ & \\
\hline $\begin{array}{l}\text { Estado nutricional } \mathbf{n} \text { (\%) } \\
\text { Riesgo de desnutrición } \\
\text { Normal } \\
\text { Sobrepeso } \\
\text { Obeso }\end{array}$ & $\begin{array}{l}1 \quad(7,1) \\
8(57,1) \\
4(28,6) \\
1 \quad(7,1)\end{array}$ & $\begin{array}{r}6(6,1) \\
38(38,4) \\
40(40,4) \\
15(15,2)\end{array}$ & $\begin{array}{l}\text { NS } \\
\text { NS } \\
\text { NS } \\
\text { NS }\end{array}$ \\
\hline $\begin{array}{l}\text { Síntomas digestivos } \mathbf{n}(\%) \\
\text { Total } \\
\text { Colon irritable } \\
\text { Constipación } \\
\text { Diarrea } \\
\text { Dolor abdominal } \\
\text { Distensión }\end{array}$ & $\begin{array}{r}35,7 \\
14,3 \\
14,3 \\
7,1 \\
21,4 \\
14,3\end{array}$ & $\begin{array}{c}42,4 \\
22,2 \\
14,1 \\
4,0 \\
31,3 \\
1\end{array}$ & $\begin{array}{l}\text { NS } \\
\text { NS } \\
\text { NS } \\
\text { NS } \\
\text { NS } \\
<0,05\end{array}$ \\
\hline Síntomas extra digestivos & 28,6 & 29,3 & NS \\
\hline Sin síntomas & 35,7 & 28,3 & NS \\
\hline
\end{tabular}


Se pesquisaron 14 familiares $(12,4 \%)$ con serología positiva, de los cuales ninguno tenía deficiencia de IgA total (Tabla 2). En los pacientes con resultados negativos, la medición de IgA/IgG-tTG identificó 1 caso adicional que, sin embargo, tenía valores de IgA dentro de los límites normales. De los 14 pacientes con IgA-tTG positiva, el EMA fue positivo en 2, ambos casos tenían valores de tTG altos (293 y $360 \mathrm{UI} / \mathrm{mL}$, respectivamente). A los 14 familiares con serología positiva se les explicó en qué consiste la enfermedad y la necesidad de realizar una biopsia de intestino delgado para confirmar el diagnóstico, pero sólo 6 aceptaron realizársela; en dos de los seis, que tenían sintomatología digestiva, pero escasa y de intensidad leve, se encontró la mucosa duodenal que se clasificó en Marsh $\geq 2$, diagnosticándose EC activa. En los otros cuatro casos la histología duodenal era normal o tenía un grado Marsh 1, por lo que les diagnosticó EC potencial, se mantuvo la dieta habitual con gluten y se les mantienen hasta ahora con controles periódicos.

\section{Discusión}

En este estudio, 14 de los 113 (12,4\%) familiares de primer grado de pacientes celíacos tenían serología positiva, lo que representa una prevalencia serológica cruda dentro del rango alto descrito en otras series extranjeras ${ }^{4-8}$. Esta cifra es más de 15 veces superior a la prevalencia serológica de EC en Chile $\mathrm{y}^{3} \mathrm{y}$ apoya fuertemente la necesidad de incorporar la búsqueda activa de la enfermedad en la consulta médica habitual, conducta que en este momento no constituye la práctica habitual en el país.

En el consenso mundial actual, el estudio serológico recomendado para hacer pesquisa de EC es IgA-tTG2, lo que también se aplica en Chile. Se ha descrito que la deficiencia de IgA es más frecuente entre los celíacos que en la población general. Sin embargo, en nuestra experiencia (datos no publicados) la medición de IgA total es una práctica infrecuente en nuestro medio, lo que claramente dificulta la interpretación de los resultados en la práctica clínica habitual. En este estudio, entre los 113 familiares de primer grado evaluados, la frecuencia de déficit alcanzó 8,0\%, cifra que coincide con los datos internacionales, es alta en comparación a lo descrito en población
Tabla 2. IgA sérica, IgA-tTG2, IgG-tTG2 y EMA en los familiares de primer grado de celíacos ya diagnosticados, distribuidos por serología positiva o negativa

\begin{tabular}{|c|c|c|}
\hline & $\begin{array}{c}\text { FPG } \\
\text { serología (+) } \\
n=14 \\
n \quad(\%)\end{array}$ & $\begin{array}{c}\text { FPG } \\
\text { serología (-) } \\
\text { n }=99 \\
\text { n }(\%)\end{array}$ \\
\hline Déficit $\lg A^{*} \mathrm{mg} / \mathrm{dL}$ & 0 & $9(9,1)$ \\
\hline TTG IgA (+) (UI/mL) & $13(92,9)$ & 0 \\
\hline TTG IgA/lgG (+) (UI/mL) & $14(100)$ & 0 \\
\hline $\operatorname{EMA}(+)$ & $2(14,3)$ & 0 \\
\hline
\end{tabular}

*Punto de corte lgA sérica: adultos $=\leq 145 \mathrm{mg} / \mathrm{dL}$; niños $=$ $\leq 21 \mathrm{mg} / \mathrm{dL}$

general y más parecida a la descrita entre celíacos. Llama la atención que fue menos frecuente de lo esperado entre los familiares con serología positiva y alta entre aquellos con serología negativa $(9,1 \%)$. Los datos del estudio no permiten explicar estos hallazgos. La medición de anti-tTG de tipo IgA e IgG concomitantemente parece útil para mejorar la búsqueda activa.

Los síntomas digestivos se observaron tanto en familiares con serología positiva como en aquellos que la tenían negativa, y en general no hubo diferencias en edad, género ni sintomatología entre estos dos grupos, sólo el síntoma distensión abdominal mostró diferencias significativas entre los individuos con serología positiva y negativa. Dado que los síntomas digestivos en general son inespecíficos, habría sido interesante analizar el papel que otras causas de ellos pudieran influir en nuestros resultados. Desgraciadamente, la calidad del grupo estudiado y los datos recogidos no permitieron evaluar el papel que pudieran jugar la obesidad o la calidad de la dieta, o componentes dietarios como por ejemplo los oligo-di-mono-sacáridos fermentables (FODMAPs), conocidos por producir distensión abdominal y otras molestias digestivas.

Uno de los problemas identificados, que al mismo tiempo representa una limitación en este estudio, es que pese a haber explicado individualmente y en detalle la necesidad de realizar una endoscopía con biopsia en todos los familiares con serología positiva, sólo 6 de ellos se la realizaron. Uno de los 14 FPG tTG positivo inició 
la dieta sin gluten cuando recibió los resultados de sus exámenes (alta tTG y EMA positivo), sin dar la oportunidad de insistir en que la biopsia intestinal era necesaria para hacer el diagnóstico y que debía realizarse antes de iniciar la dieta. La sesión de educación analizó con ellos también los pros y contras de instaurar tratamiento con dieta sin gluten cuando se sufre una variante poco sintomática, y los potenciales riesgos a largo plazo. Estos individuos tenían relativamente pocas molestias y estas eran de intensidad baja, por lo que las aceptaban como "su manera de ser". Creemos que este factor podría explicar, al menos en parte, su reticencia a completar el estudio. Aunque esta especulación fuera sólo parcialmente correcta, sugiere que el equipo médico debiera ser capacitado para enfrentar el proceso diagnóstico según los criterios actuales definidos por ESPGHAN 2012; debiéramos construir un protocolo de acción definido, que por un lado favorezca la búsqueda activa, y por otro capacite al equipo de salud en educar a las familias acerca de la necesidad de contar con el diagnóstico de certeza.

Un comentario aparte merece la llamada enfermedad celíaca potencial. Los hallazgos histológicos en la biopsia duodenal no sólo confirman el diagnóstico, también definen la terapia, pues aquellos con alteraciones mínimas o sin alteraciones (Marsh 0-1), los celíacos potenciales, no tienen indicación de dieta sin gluten, sino que deben permanecer en seguimiento. En nuestra serie, 4 pacientes fueron catalogados como EC potencial, se les explicó lo que esta significa, y han permanecido con régimen completo, controlándose periódicamente según la evolución de la sintomatología clínica y la serología.

Con estos resultados, se concluye que la prevalencia serológica cruda mediante tTG2 es alta $(12,4 \%)$ en los FPG de pacientes celíacos estudiados, más de 10 veces mayor que la que se describe en población general chilena, y comparable a cifras descritas internacionalmente. La búsqueda activa en los familiares de celíacos debe implementarse en la práctica médica habitual, a nivel nacional, según los definen las diversas guías actualmente disponibles. En general, ellas coinciden en que se debe hacer el rastreo serológico en los pacientes de riesgo, independiente de si presenten o no sintomatología, y de la intensidad de los síntomas que presenten. Al mismo tiempo, es necesario educar y motivar a los pacientes pesquisados sobre la importancia de completar el estudio con biopsias duodenales obtenidas mediante endoscopía, para confirmar el diagnóstico. Sin duda, hacerlo mejoraría la posibilidad de un mejor diagnóstico y manejo de la enfermedad en el largo plazo, con menores costos y mejor calidad de vida para el paciente.

\section{Referencias}

1. Husby S, Koletzko S, Korponay-Szabo IR, Mearin ML, Phillips A, Shamir R, et al. European Society for Pediatric Gastroenterology, Hepatology, and Nutrition guidelines for the diagnosis of coeliac disease. J Pediatr Gastroenterol Nutr 2012; 54 (1): 136-60.

2. Kang JY, Kang AH, Green A, Gwee KA, Ho KY. Systematic review: worldwide variation in the frequency of coeliac disease and changes over time. Aliment Pharmacol Ther 2013; 38 (3): 226-45.

3. Hill I, Fasano A, Schwartz R, Counts D, Glock M, Horvath $\mathrm{K}$. The prevalence of celiac disease in at-risk groups of children in the United States. J Pediatr 2000; 136 (1): 86-90.

4. Chile MdS. www.redsalud.gov.cl/portal/url/.../99bbf09a908d3eb8e04001011f014b49. 2009.

5. Murray JA, Van Dyke C, Plevak MF, Dierkhising RA, Zinsmeister AR, Melton LJ, 3rd. Trends in the identification and clinical features of celiac disease in a North American community, 1950-2001. Clin Gastroenterol Hepatol 2003; 1 (1): 19-27.

6. Fasano A, Catassi C. Clinical practice. Celiac disease. N Engl J Med. 2012; 367 (25): 2419-26.

7. Gardiner AJ MK, Walker-Smith JA. A family study of coeliac disease. Aust Paediatr J 1973; 9: 18-24.

8. Dowd B W-SJ. Samuel Gee, Aretaeus and the coeliac disease. Brit Med J 1974; 2: 45-7.

9. Dicke WK, Weijers HA, Van De Kamer JH. Coeliac disease. II. The presence in wheat of a factor having a deleterious effect in cases of coeliac disease. Acta Paediatr 1953; 42 (1): 34-42.

10. Araya M, Mondragón A, Pérez-Bravo F, Roessler JL, Alarcón T, Ríos G, et al. Celiac disease in a Chilean population carrying Amerindian traits. J Pediatr Gastroenterol Nutr. 2000; 31 (4): 381-6.

11. Canales RP, Araya QM, Alliende GF, Hunter MB, Alarcón OT, Chávez SE. [Diagnosis and clinical presentations of celiac disease: a multicenter study]. Rev Med Chile 2008; 136 (3): 296-303.

12. Troncone R, Discepolo V. Celiac disease and autoimmunity. J Pediatr Gastroenterol Nutr 2014; 59 Suppl 1: S9-S11. 
13. Verbeke S, Cruchet S, Gotteland M, Ríos G, Hunter B, Chávez E, et al. [Risk markers for insulin-dependent diabetes mellitus and duration of exposure to gluten in celiac patients]. Rev Med Chile 2004; 132 (8): 97984.

14. Botero-López JE, Araya M, Parada A, Méndez MA, Pizarro F, Espinosa N, et al. Micronutrient deficiencies in patients with typical and atypical celiac disease. J Pediatr Gastroenterol Nutr 2011; 53 (3): 265-70.

15. Somech R, Spirer Z. Celiac disease: extraintestinal manifestations, associated diseases, and complications. Adv Pediatr 2002; 49: 191-201.

16. Maki M, Holm K, Lipsanen V, Hallstrom O, Viander $\mathrm{M}$, Collin $\mathrm{P}$, et al. Serological markers and HLA genes among healthy first-degree relatives of patients with coeliac disease. Lancet 1991; 338 (8779): 1350-3.

17. Fasano A, Berti I, Gerarduzzi T, Not T, Colletti RB, Drago S, et al. Prevalence of celiac disease in at-risk and not-at-risk groups in the United States: a large multicenter study. Arch Intern Med 2003; 163 (3): 286-92.

18. Mustalahti K, Sulkanen S, Holopainen P, Laurila K, Collin P, Partanen J, et al. Coeliac disease among healthy members of multiple case coeliac disease families. Scand J Gastroenterol 2002; 37 (2): 161-5.

19. Dube C, Rostom A, Sy R, Cranney A, Saloojee N, Garritty $\mathrm{C}$, et al. The prevalence of celiac disease in average-risk and at-risk Western European populations: a systematic review. Gastroenterology 2005; 128 (4 Suppl 1): S57-67.

20. Biagi F, Corazza GR. First-degree relatives of celiac patients: are they at an increased risk of developing celiac disease? J Clin Gastroenterol 2009; 43 (1): 3-4.

21. López-Hoyos M, Bartolomé-Pacheco MJ, Castro B, Fernández F, de las Heras Castano G. [Screening of celiac disease in first-degree relatives]. Med Clin (Barc). 2003; 120 (4): 132-4.
22. Karinen $H$, Karkkainen $P$, Pihlajamaki J, Janatuinen E, Heikkinen M, Julkunen R, et al. HLA genotyping is useful in the evaluation of the risk for coeliac disease in the 1st-degree relatives of patients with coeliac disease. Scand J Gastroenterol 2006; 41 (11): 1299-304.

23. Long KH, Rubio-Tapia A, Wagie AE, Melton LJ, 3rd, Lahr BD, Van Dyke CT, et al. The economics of coeliac disease: a population-based study. Aliment Pharmacol Ther 2010; 32 (2): 261-9.

24. Byass $\mathrm{P}$, Kahn K, Ivarsson A. The global burden of childhood coeliac disease: a neglected component of diarrhoeal mortality? PLoS One. 2011; 6 (7): e22774.

25. Corrao G, Corazza GR, Bagnardi V, Brusco G, Ciacci C, Cottone M, et al. Mortality in patients with coeliac disease and their relatives: a cohort study. Lancet 2001; 358 (9279): 356-61.

26. Rubio-Tapia A, Kyle RA, Kaplan EL, Johnson DR, Page W, Erdtmann F, et al. Increased prevalence and mortality in undiagnosed celiac disease. Gastroenterology 2009; 137 (1): 88-93.

27. Conrad K, Roggenbuck D, Ittenson A, Reinhold D, Buettner T, Laass MW. A new dot immunoassay for simultaneous detection of celiac specific antibodies and IgA-deficiency. Clinical chemistry and laboratory medicine: CCLM/FESCC 2012; 50 (2): 337-43.

28. McGowan KE, Lyon ME, Butzner JD. Celiac disease and IgA deficiency: complications of serological testing approaches encountered in the clinic. Clin Chem 2008; 54 (7): 1203-9.

29. Rubio-Tapia A, Hill ID, Kelly CP, Calderwood AH, Murray JA, American College of G. ACG clinical guidelines: diagnosis and management of celiac disease. Am J Gastroenterol 2013; 108 (5): 656-76; quiz 77.

30. Hill P, Austin A, Forsyth J, Holmes G. British Society of Gastroenterology guidelines on the diagnosis and management of coeliac disease. Gut 2014 Oct 9. 